\title{
Cultura forestal en Quintana Roo, México; observaciones y perspectivas
}

\author{
Michael Robert Keyes Hennin ${ }^{1}$
}

\begin{abstract}
RESUMEN
La etnia maya en Quintana Roo es la heredera de la civilización más sobresaliente de las que se han desarrollado en la selva tropical. Sus conocimientos autóctonos acerca del medio ambiente siguen vigentes y sirven como caso ejemplar de una "cultura forestal". Un análisis histórico indica la concepción que tuvieron sobre sus recursos forestales, y cómo cambiaron su valorización ante la presencia de otras culturas sin que se haya dejado al margen a la cultura autóctona. Actualmente, esta cultura se reestructura para incorporar elementos contemporáneos de acuerdo con el mercado, y como parte de la inevitable transculturación. Las experiencias de las instituciones, gubernamentales y no gubernamentales, coinciden en sus metas y programas con los mayas de la Península de Yucatán, rebasando ya la silvicultura comunitaria y formulando un ejemplo que podemos llamar etno-silvicultura. La educación resulta ser una crucial incógnita que debe ser atendida para que la sociedad evolucione y mejore su calidad de vida.
\end{abstract}

PALABRAS CLAVE

Silvicultura comunitaria, manejo forestal tropical, etno-silvicultura.

\begin{abstract}
The ethnic Mayan inherited one of the most outstanding civilizations which have developed in tropical forests. Their autochthonous knowledge regarding the environment continues to serve today as an exemplary case of "forest culture." A historical analysis reveals the conception which these ethnics had regarding their forest resource and how this valorization has changed in the presence of other cultures without brushing aside their autochthonous culture. Presently this culture is restructuring to incorporate contemporary elements of the global market as parte of their inevitable transculturation. The experiences of the government and non-government institutions coincide in their programs and goals with the Mayan's on the Yucatan Peninsula, now surpassing community forestry and are formulating an example of what we can call ethno-silviculture. Education remains as a critical unknown factor which should be attended to enable the continued evolution of the society towards the improvement of the quality of life.
\end{abstract}

KEY WORDS

Community forestry, tropical forest management, ethno-silviculture. 


\section{INTRODUCCION}

Uno de los mayores retos de la silvicultura tropical es el de comprender las interrelaciones entre las diversas actividades antropogénicas en la selva y el desarrollo de las áreas más marginadas del planeta. El desarrollo económico de estas regiones geográficas, que casi siempre corresponde a los llamados países del tercer mundo, ha sido una preocupación constante durante el presente siglo (Aguirre, 1970, 1973). Un importante sector intelectual llamó la atención sobre la suerte de estos países subdesarrollados, e hizo suponer que el modelo occidental de desarrollo era apropiado. Las desventajas podrían ser compensadas, si el acervo de técnicas y conocimientos, descubiertos básicamente por los países europeos fueran puestos a disposición de las naciones del tercer mundo. Posteriormente, sin embargo, en los intentos iniciales de esta idea se pudo ver que el modelo occidental no podía generalizarse.

El explosivo crecimiento de la población en los países subdesarrollados obligaba a cambiar totalmente los términos de referencia (Aguirre, 1973). El despojo y la degradación de los recursos naturales que acompaña esta acelerada y violenta integración del indio Americano al desarrollo moderno es no solamente el terreno de acción indigenista, sino también de los forestales. Herskovits (1987) ha señalado que estudios de contacto (de culturas) deben realizarse dondequiera que "los conduzcan los materiales y éstos tienen el hábito de desdeñar las barreras intelectuales que separan los campos disciplinarios". Con respecto a la silvicultura tropical, ya han pasado dos décadas desde que se ha llamado la atención a los científicos para que se tome en cuenta la participación de la comunidad en su planeación forestal (Eckholm, 1979). Asimismo, ha sido señalado en foros internacionales que la falta de participación comunitaria puede limitar el potencial de movilización para el apoyo público en favor de la conservación (TED, 1993).

\section{EL CONCEPTO DE LA CULTURA FORESTAL Y LA TRANSCULTURACION}

Para entender a una etnia y sus recursos forestales, hay que revisar antes lo que históricamente se ha entendido como cultura. La cultura, como definición tiene el sentido de desarrollar y acumular costumbres; son actitudes, actividades y creencias en una sociedad dada. Desde el punto de vista antropológico, la cultura es todo lo que el hombre crea de una forma tangible o abstracta. Herskovits (1987) nos señala que aunque existen muchas definiciones de "cultura", todas coinciden en que esta es aprendida y que le permite al hombre adaptarse a su ambiente natural; aunque como definición útil menciona la siguiente: "Cultura es la parte del ambiente hecha por el hombre". El cómo se percibe lo que es la cultura resulta ser el espejo de cada sociedad.

Por otro lado, la transculturación se ha definido como el "intento de liquidar o al menos negar, la cultura autóctona" (Varela, 1985). El empeño de la transculturación se encuentra con que es el negativo de un trabajo de servidumbre económica, hasta biológica (Fanon, 1973). Paralelamente a este concepto, se encuentra el de la invasión cultural. Esta consiste en la penetración que hacen los invasores en el contexto cultural de los invadidos, imponiendo a éstos su visión del mundo, en la medida misma en que frena su creatividad, inhibiendo su expansión (Freire, 1970).

En el encuentro entre diferentes culturas, que tienen diferentes escalas de valores, se produce pérdida de valores, revalorizaciones y pérdida de conocimientos autóctonos con la transculturación. Mediante la imposición cultural sistemática con diferente impacto 
en los diversos aspectos culturales de una sociedad, la fusión de culturas modifica la escala de valores implicando, por supuesto, a los recursos naturales (Aguirre, 1970). Surge entonces la pregunta ¿cómo llamar lo que va en contra y degrada la calidad de vida de la comunidad y las cualidades que promulga una cultura a "favor" de otra? Entonces se establece una lucha que tiene especial relevancia en los conceptos ecológicos y en los impactos de las actividades culturales y contra-culturales que se realizan en las selvas.

\section{LA ETNIA MAYA DE QUINTANA ROO}

En América Latina no son muchos los ejemplos de éxitos económicos en la colonización de tierras tropicales. Por el contrario, la ambición provocó la caída de los más hábiles exploradores y conquistadores. Lo inhóspito que resulta el trópico-húmedo para los forasteros está documentado en el relato de Díaz del Castillo (1986) acerca del viaje de Cortés para castigar a Cristóbal de Olid y en los relatos del sufrido viaje de Alonso Dávila, cuando pasó precisamente por las provincias de Uamil y Chetumal, antes de 1532 (Gobierno del Estado de Quintana Roo, 1984). Si bien no prosperaron los europeos como planearon, su dominio fue efectivo al paso del tiempo. El encuentro entre Mayas y europeos fue exitoso, especialmente en las costas y riberas en donde ya existía el intercambio comercial. El dominio eventual de estos sitios estratégicos fluviales, dio lugar a que el comercio preestablecido quedara en manos de los conquistadores. El europeo, carente de alternativas agropecuarias para las regiones tropicales en general, explotaba el capital en las selvas (Foster, 1987) como alternativa casi única.

En el ámbito de interés para este artículo, es más que aparente que lo que más dificulta el entendimiento de la cultura forestal es la desaparición de las etnias que la crearon y poseyeron durante siglos. La civilización Maya Clásica floreció y sufrió un colapso entre los años 650 y 900 D.C., mucho antes que llegaron los españoles. Los factores que involucraron el abandono de sus grandes centros culturales han sido motivo de diversos estudios durante el presente siglo (Cooke, 1931; Abrams y Rue, 1988). Lo que es indiscutible es que la cultura maya no tiene paralelo entre las grandes civilizaciones tropicales; una de las razones es el hecho de haberse desarrollado, comparativamente, en un ambiente físico poco favorable para la agricultura (Morley, 1981). Algunos investigadores sostienen la hipótesis de que la tala inmoderada de los recursos forestales dañó seriamente la fertilidad de los suelos, contribuyendo al colapso de su infraestructura agrícola y al abandono consecuente de sus ciudades (ver Abrams y Rue, 1988).

Otras investigaciones proponen otra perspectiva: Barrera et al (1977) y Gómez ( $\sin$ fecha) mencionan que las técnicas utilizadas por los mayas probablemente desempeñaron un papel muy importante en la domesticación o semi-domesticación de muchos elementos de su flora y fauna. Algunos de sus elementos son evidentes hasta hoy: en algunas de sus milpas dejan franjas de vegetación natural arbórea (tol che), y bardas de piedras (pet kot), mismos que con el tiempo y abandono forman parches de selvas con especies dirigidas (Gómez et al., 1987). En todo caso, la cultura forestal maya comprende la suma de las experiencias de cada hombre trabajando con su tecnología, determinando que, dónde, cómo y cuánto ocurre una alteración en la flora y la fauna que conforman la selva. Si bien las técnicas pasadas no constituyen la "silvicultura" como se conoce a la fecha, actualmente no son menos importantes que las técnicas modernas que hoy se practican. 
Los mayas de Quintana Roo no han estado históricamente sometidos como otras etnias. Por el contrario, en Quintana Roo se establecieron los grupos mayas que decidieron refugiarse en la selva para evitar ser sometidos. Tal aislamiento les permitió mantener mayor cohesión cultural, posiblemente apoyados en lo que menciona Aguirre (1973) logrando cambios de forma y no de fondo, es decir, conservando sin cambio sus elementos fundamentales y modificando únicamente su apariencia externa. A través de la convivencia y de la historia, se puede conocer mejor lo que es y ha sido la cultura forestal para los mayas de Quintana Roo y su relación, precisamente, con los recursos forestales.

Lo adecuado al medio natural de Quintana Roo ha sido y sigue siendo el modo de vida de la civilización maya; ésta ha podido prosperar y perdurar en un ambiente selvático. Barrera et al. (1977) mencionan que la composición florística actual de las selvas de la región refleja el uso tradicional, aún después de cinco siglos del quebrantamiento de su continuidad cultural. Los mayas integraron un sistema de nomenclatura y taxonomía cuyos restos todavía perduran (Flores y Ucán, 1983), y posiblemente propiciaron con su manejo una alta densidad de árboles forrajeros, frutales y resinosos. Existe entonces la posibilidad de que la actual alta densidad específica de árboles como Annona spp. Chrysophyllum mexicanum, Spondias mombin, Psidium guajava, Brosimum alicastrum, Manilkara zapota y Pouteria campechiana, se deba a relictos de la etnoflora que mantenían los mayas antiguamente en sus huertos familiares (Gómez, sin fecha).

\section{PERIODO COLONIAL E INFLUENCIA INGLESA}

Menos apreciadas son las bases

para que los Mayas iniciaran la comercialización del recurso forestal con las potencias europeas. Desde el siglo XVII hubo campamentos para la extracción del palo de Campeche. Pero en los siglos XVII y XVIII, naves provenientes de la Habana y otros puertos recorrieron el Golfo y la Península en busca de caoba. También la madera de los mayas fue importante entre 1720 y 1830 para Inglaterra (Lamb, 1966), sin que hubiera inversiones por su parte para que estas explotaciones impulsaran el desarrollo local. El comercio de maderas tintóreas y preciosas que se llevó a cabo en forma clandestina en la costa oriental y en la ribera del Río Hondo hasta fines del siglo XIX. En 1898, México determinó poner fin a la piratería inglesa y envió fuerzas militares a la Bahía de Chetumal para impedir explotaciones ilegales en las selvas Quintanarroenses (Medina, 1948).

El comercio entre ingleses y mayas no solamente proveyó de armas a los nativos, sino también de ideologías y entrenamiento moderno con materiales de consumo y bélicos. En el seno de la resistencia se originó el culto de la "cruz parlante" en tres caobas, representando la libertad de expresión, religiosa y de comunicación militar para los rebeldes mayas de Quintana Roo. Durante la llamada "Guerra de Castas" entre 1847 y 1901, los británicos, apoyados por los mayas, realizaron la explotación maderera en los dos lados del Río Hondo. Así, los mayas lucharon y retuvieron su cultura mediante la explotación de sus recursos forestales a cambio de armas, pólvora y municiones. Hasta hoy, existe entre los mayas de Quintana Roo, el mismo orden militar que se manifiesta abiertamente al lado de los gobernantes actuales en los actos cívicos.

\section{PERIODO POST-INDEPENDENCIA}

El General Antonio López de Santa Ana dio a conocer el chicle a James Adams, un norteamericano que llegó a Tuxpan, para valorar la posibilidad de su 
explotación. Pronto se dieron cuenta que la cantidad de chicozapotes en la península de Yucatán era superior a la que había en la vertiente del Golfo. En la península se llegaron a "picar" entre siete y trece árboles por hectárea durante el auge de la exportación de chicle (Márquez, 1943). Para iniciar la actividad económica en Quintana Roo, se creo una colonia penal, por lo que se construyó la vía angosta entre Felipe Carrillo Puerto y Vigía Chico apoyando así la exportación del chicle. Fue la misma actividad chiclera conducida por los caciques, como el General May, que de alguna manera sirvió para "pacificar" a los indígenas, principalmente mediante licor, mujeres y víveres.

A pesar del incipiente intercambio comercial con los españoles, el odio y el miedo de los "salvajes" se convirtió en su desprestigio en el este de la península. Por ejemplo, en el siglo pasado, para justificar la ampliación de la frontera agropecuaria y el despojo de los mayas en Quintana Roo, se expresó claramente lo siguiente: “... deseamos todo lo que conduce al avance de la humanidad (...) y que los salvajes que existen en la Península (...) también deben adquirir los beneficios de la civilización" (Diario Oficial de Campeche, 13 de marzo, 1891; tomado de Konrad, 1998).

\section{EPOCA MODERNA}

Al inicio del siglo, en México se propició una apertura comercial durante la época de Porfirio Díaz. Se inició así la transición de una sociedad rural y preindustrial hacia una nación moderna, mediante una creciente infraestructura, la extracción sistemática y la exportación. Sin embargo, la época se caracteriza más como económico-explotadora y simbólica respecto a la conservación. En 1909 el Banco Nacional de México expidió
400,000 dólares para explotar más de $1,036,000$ ha de palo de tinte y chicle en el nordeste de la Península. El área contaba con 21 elementos de personal forestal, de los cuales solamente un subinspector y dos guardabosques fueron comisionados en el entonces Territorio de Quintana Roo. Sin embargo, por los ataques de "resistencia" maya, las enfermedades y la mala alimentación de los trabajadores, se terminó con la aventura antes de alcanzar 3,900 ha (SEP, 1985). De todas maneras, a principios de este siglo, los mayas de la península estaban trabajando integrándose hacia la sociedad moderna.

La industrialización de los lugares más remotos se fomentó desde la época del Porfiriato, mediante concesiones de grandes extensiones de selvas para las inversiones madereras, también se favoreció la infraestructura portuaria, vial, e industrial (Konrad, 1998). Los primeros permisos para la explotación se otorgaron por medio de contratos-concesiones a compañías como Mengel Brothers and Co., por superficies que comprendían entre 5,000 y 100,000 hectáreas.

Fue Lázaro Cárdenas del Río quién vio la importancia del chicle como actividad económico-social para los nativos. Posteriormente, formuló el reparto agrario a los "ejidos forestales" de grandes extensiones en Quintana Roo. Las parcelas comunes eran de 420 ha per cápita, extensión que más o menos se ha conservado hasta décadas recientes en la entidad. Otro paso fundamental fue la creación del cooperativismo en el estado, promovido y concretado por el General Melgar (Rosado, 1940). Así, el cambio de un órgano de control y vigilancia tradicional hacia la promoción y el apoyo al desarrollo de las empresas forestales campesinas se ha venido logrando poco a poco durante el presente siglo. 


\section{EPOCA CONTEMPORANEA}

En la década de 1950 se dieron concesiones a la empresa Maderas Industrializadas de Quintana Roo (MIQRO). Esta tenía una concesión de 540,000 hectáreas y tipificaba el estilo maderero; tenía planes de ordenación de las áreas de reciente colonización en cada ejido. El esquema, a pesar de que era desigual para los campesinos, contribuyó a evitar y amortiguar los cambios del uso de la tierra para fines agropecuarios. Además, MIQRO sí realizaba reforestación, pero pagaba solamente el concepto de "derecho de monte" a los poseedores del bosque y dio poca continuidad a las plantaciones establecidas (Argüelles, 1991). Una de las ventajas obtenidas de los trabajos de MIQRO fue que los ejidatarios se acostumbraron a trabajar la madera y trabajar sobre una planeación. Al terminar la concesión de MIQRO, en los inicios de la década de los ochenta, se produjo una transición de los poderes hacia una nueva página en la cultura forestal de la región.

\section{PLAN PILOTO FORESTAL}

En 1982 se respaldó la producción a través de un grupo denominado Plan Piloto Forestal de Quintana Roo (PPF) integrado por técnicos forestales mexicanos y algunos provenientes de la Gesellschaft für Technische Zusammenarbeit (GTZ) de Alemania. El PPF fue concebido bajo el Acuerdo México-Alemania, y en este marco, la Secretaría de Agricultura y Recursos Hidráulicos colaboró con la participación de investigadores y el préstamo de los vehículos de sus campos experimentales durante la etapa de arranque. El grupo técnico del PPF decidió abandonar su papel pasivo de burócratas y se convirtió en sujeto activo para el entrenamiento de los campesinos participantes del manejo forestal tropical. El PPF funge como un organismo de promoción y apoyo al desarrollo de las empresas forestales campesinas y no como organismo de control y vigilancia (Galletti y Argüelles, 1987). Al finalizar una década de trabajo, los poseedores del recurso ya contaban con capacitación para realizar inventarios forestales, selección de arbolado para derribar (marqueo), extracción de trozas y administración de su propia empresa forestal. Parte de la capacitación campesina se basa en introducir los conceptos elementales de la silvicultura, tales como inventario, ciclo de corta, diámetros mínimos y protección forestal. Para una adecuada administración se procedió a enseñar diversas técnicas de estimación de volúmenes de trozas cortadas, así como llevar control y contabilidad básicos. Se puso énfasis en la necesidad de diversificar el aprovechamiento hacia otras especies por razones económicas y ecológicas (Argüelles, 1991).

Bajo el manejo forestal actual del PPF se intenta cortar dos metros cúbicos de madera blanda o dura tropical por cada metro cúbico de caoba o cedro y el ciclo de corte consiste en intervenciones cada 25 años. Los ejidos utilizan la regeneración natural como medida principal para repoblar áreas cortadas. Este sistema de manejo propicia el establecimiento y crecimiento de especies deseables por la perturbación creada (Macario, 1991; Argüelles, 1991). Por lo menos ocho especies comerciales existen en abundancia y hasta prosperan después de las perturbaciones más comunes (Snook, 1993); no obstante, la misma autora confirmó lo mencionado por Juárez (1988) al inferir que la caoba necesita hasta 120 años para alcanzar los $55 \mathrm{~cm}$ de diámetro para el corte comercial. La regeneración, natural o artificial no ha prosperado como se esperaba.

Inicialmente, los ingresos per cápita aumentaron en los ejidos participantes. Aunque los rendimientos netos por la explotación de la selvas son inferiores a 
$\$ 10 \mathrm{dl} / \mathrm{ha} /$ año. Esto significa un promedio de ingresos a los ejidatarios de $\$ 1,813$ $\mathrm{dl} /$ hombre en el mejor ejido (Richards, 1993). Actualmente, los ingresos por venta de madera dependen tanto de las existencias volumétricas de unas 18 especies comerciales así como de las otras actividades económicas, las cuales varían según el ejido. Por ejemplo, en la Zona Maya los ingresos por venta de la producción milpera constituyó el 19\% y del corte de madera constituyó solamente el $4 \%$ de las ventas (Murphy, 1992). No obstante lo anterior, los ingresos maderables alcanzaron más del $49 \%$ si se considera la errática producción por concepto de durmientes para ferrocarril.

\section{RESERVAS FORESTALES ESTRATEGICAS Y DE LA BIOSFERA}

Las bases para el manejo sustentable del bosque son las Reservas Forestales Estratégicas (RFE) que ya suman a más de 400,000 ha (Richards, 1991). No se permite hacer milpas ni realizar otras actividades agropecuarias en la RFE, conteniéndose así la marcha de los desmontes. Los técnicos apoyan en las labores de inventario y en la consecución de los permisos para explotar estos rodales. Una vez establecidas, las RFE son parte de la planeación en cada ejido. Para la consolidación de la nueva cultura forestal son requisitos importantísimos la planeación y la limitación de las actividades que perjudican a las selvas; asimismo se debe considerar la puesta en práctica de elementos modernos en el manejo forestal. Además de las RFE, el estado de Quintana Roo cuenta con casi 530,000 ha en la Reserva de la Biósfera Sian Ka'an (SEDESOL, 1993), misma que a su vez forma un bloque de selvas y humedales contiguos en la parte central de la entidad.

\section{MANEJO FORESTAL CAMPESINO}

Actualmente, los recursos forestales ya están en manos de campesinos mexicanos después de diez años de trabajo continuo. Muchos académicos nacionales e internacionales forman parte de la base científica para re-dirigir las actividades hacia aspectos sociales, económicos y ecológicos de interés nacional. La conciencia de los campesinos sobre los cuidados de sus recursos abarca ahora rubros inesperados. Por ejemplo, los ejidatarios son muy eficientes en la vigilancia de intrusos en áreas de aprovechamiento. Los transgresores de las normas dentro de los ejidos pueden perder utilidades provenientes de la madera si realizan actividades que perjudiquen la sustentabilidad de los aprovechamientos. Hay casos, en algunos ejidos, de intrusos que han visto confiscados sus equipos y que incluso fueron encarcelados. Efectivamente se ha escrito un capítulo nuevo respecto a las relaciones entre la utilización y la sustentabilidad de la selva en forma práctica.

Al terminar el dominio de los industriales sobre el recurso forestal en el sur de Quintana Roo, surge la época actual de participación campesina en la misma zona, que luego se extiende hacia el norte y el oeste. Por fin los mayas del este de la península están básicamente por encima de los intermediarios y la cultura forestal que se ha forjado con los siglos, empieza a entrar en la transformación de aserraderos, talleres y artículos terminados, consolidándose, diversificándose y también se reforma para el nuevo mercado.

Cabe resaltar que en el estado de Quintana Roo existen factores únicos que han favorecido el buen desarrollo de una cultura forestal constante. Entre los más importantes podemos mencionar: ser el estado más joven de la República, permitiéndole mantenerse al margen de explotaciones intensivas por presiones 
económicas; tener el más bajo índice de población del país, facilitando el acercamiento entre funcionarios gubernamentales y campesinos y que las existencias relativas de maderas preciosas por hectárea, así como sus incrementos volumétricos, son más altas que en otras partes del trópico mexicano.

Por otro lado, hay todavía deficiencias fundamentales en la transmisión de los conocimientos. Si se está perdiendo la sabiduría tradicional, debe remediarse promoviendo la tradición oral incluso en su propia lengua (Maya no escrito). Por otra parte, en la perspectiva de una silvicultura moderna se hace necesario revisar los planes de estudio de las carreras forestales en México para dar un mejor y más real acercamiento a los problemas no solo de producción sino también y muy especialmente a los de conservación de bosques y selvas mediante producción sostenible a largo plazo (Keyes, 1995). Salvo el Instituto Tecnológico Agropecuario No. 16 de Juan Sarabia, Quintana Roo, que esporádicamente da una cátedra en silvicultura, el trópico mexicano no cuenta con educación forestal formal para asegurar que la administración del recurso forestal quede en manos de los campesinos a largo plazo. La Universidad de Quintana Roo está iniciando también un programa Forestal.

\section{GESTION INSTITUCIONAL}

La debilidad generalizada de las instituciones federales ante los protagonistas de la época ilustra lo incipiente de la educación y de otros programas oficiales. Lamentablemente, todavía existe una deficiente ética profesional. Esta situación no se ve favorecida por el esquema actual de asesoría técnica que se cobra por metro cúbico de madera autorizada. Para eliminar el sometimiento, la corrupción y los abusos, se requiere propiciar el acceso de los campesinos a la tecnología y a los conocimientos que específicamente puedan apoyar su propia evolución cultural y económica. Si por silvicultura se entiende la ciencia y el arte de cultivar el bosque, entonces hay todavía mucho trabajo por hacer en la silvicultura tropical en general y mucho por mejorar en Quintana Roo en particular. Lo anterior, aunado a la falta de vigilancia sobre el cumplimiento de las leyes, hace casi inevitable que se generen relaciones oscuras entre funcionarios públicos 0 militares y los madereros saqueadores del recurso que son la base del lastimoso intermediarismo.

El trabajo que se ha hecho en las selvas aún no es suficiente. El desarrollo sustentable constituye un reto tanto conceptual como concreto. Para los que forman parte del gremio forestal en México, la cultura forestal debe dejar de ser un negocio no sustentable, debe ser un reto y una meta que se acerque más a la esencia de la vida misma.

\section{CONCLUSIONES}

La cultura maya de Quintana Roo es uno de los mejores ejemplos de culturas tropicales con larga historia y que sigue viva en el territorio mexicano. Su más reciente capítulo tiene gran relevancia como modelo de organización participativa susceptible de ser aplicado en otras selvas tropicales. El modelo de Plan Piloto Forestal ha incorporado conocimientos de la etnia maya de Quintana Roo sobre el medio ambiente y sigue evolucionando como parte de la transculturación que se reestructura para incorporar los elementos contemporáneos de la globalización de mercado. Sin duda, la capacitación técnica ha jugado un papel primordial para lograr la integración de elementos diversos para resolver los planteamientos modernos. Sin embargo, hay que señalar que todavía hace falta poner mayor énfasis en la educación formal para que la 
cultura forestal alcance la importancia que requiere para ser parte positiva y real del desarrollo productivo y sustentable.

También se requiere de la dedicación y de la voluntad profesional para vigilar y fomentar que las condiciones sean justas para cada caso de las diferentes sociedades autóctonas del país. Se debe asegurar que los legítimos poseedores del recurso forestal tengan, por lo menos, la capacidad de participar en los acuerdos para lograr el beneficio social a través del intercambio comercial justo y equilibrado y sobre todo, para lograr la preservación de las selvas.

La sociedad mexicana se encuentra actualmente en un cruce de caminos. Al ver el ejemplo de los mayas que sirve como base histórica para el planteamiento de una cultura forestal propia y moderna a 500 años del inicio del choque entre dos culturas, se presenta entonces una tarea para cada uno de los que trabajan en el gremio forestal. Será necesario reconocer, respetar y dar lugar a la participación activa del conocimiento étnico mediante la integración de sus conceptos etnosilvícolas para la nueva cultura forestal.

\section{RECONOCIMIENTOS}

Expreso mi más profundo reconocimiento a los revisores anónimos cuyas opiniones contribuyeron de manera sustancial a la forma final de este manuscrito. De igual manera, los valiosos comentarios y sugerencias del Dr. Raymundo Dávalos Sotelo con respecto a la organización del artículo son ampliamente agradecidos. La realización de este manuscrito se hizo utilizando fondos fiscales asignados al Departamento de Productos Forestales y Conservación de Bosques con la clave 902-13.

\section{REFERENCIAS}

Abrams, E. y D. Rue. 1988. The causes and consequences of deforestation among the prehistoric Maya. Human Ecology 16 (4): 377-395.

Argüelles, A. 1991. Experiencias en desarrollo rural: El caso del Plan Piloto Forestal de Quintana Roo, México. In: D.V. Johnson, ed. Proceedings of the Humid Tropical Lowlands Conference: Development Strategies and Natural Forest Management. Vol. 3. Bethesda, Maryland. EUA:1-14

Aguirre B.,G. 1970. El proceso de aculturación y el cambio sociocultural en México. Instituto de Ciencias Sociales. México, D.F. 205 p.

Aguirre B.,G. 1973. Regiones de refugio. Instituto Nacional Indigenista, Mexico, D.F. 366 p.

Barrera, A., A. Gómez P. y C. Váquez Y. 1977. El manejo de las selvas por Ios Mayas: sus implicaciones silvícolas y agrícolas. Biótica 2 (2):47-61.

Cooke, C. 1931. Why the Maya cities of the Peten District, Guatemala, were abandoned. Journal of the Washington Academy of Sciences 21 (13):283-287.

Díaz del Bastillo, B. 1986. La historia de la conquista de la Nueva España. Colecc. Sepan Cuantos No.5. Ed. Porrúa, México, D.F.

Eckholm, E. 1979. Forestry for human needs. Interciencia, Julio-Agosto. 207-214. 
Fanon, F. 1973. Por la revolución africana (traducción de Demetrio Aguilera Malta). Fondo de Cultura Económica. México, D.F.

Flores, J. y E. Ucan. 1983. Nombres usados por los Mayas para designar a la vegetación. Cuadernos de divulgación No. 10. Instituto Nacional de Investigaciones sobre Recursos Bióticos. Xalapa, Ver. México. 30 p.

Foster, B. 1987. The Baymen's legacy. Cubola Productions. Belize, Centro América.

Freire, P. 1970. Pedagogía del oprimido (traducción de Jorge Mellado). Siglo XXI Editores. México, D.F.

Galletti, H. y A. Argüelles. 1987. La experiencia en el aprovechamiento de las selvas en el estado de Quintana Roo, México. "Del modelo forestal clásico a un modelo forestal alternativo". Taller Internacional sobre Silvicultura y Manejo de Selvas, SARH, 11 a 20 de Mayo; Chetumal, Q.R. México.

Gobierno del Estado de Quintana Roo. 1984. Lecturas básicas para la historia de Quintana Roo. Antología. Descubrimiento y conquista. pp. 11-129. Tomo II. Chetumal, Quintana Roo, México.

Gómez P., A., J. Salvador y V. Sosa. 1987. The "pet kot": a man-made tropical forest of the Maya. Interciencia, Jan-feb:10-15.

Gómez P., A. Sin fecha (circa 1995). La Silvicultura maya. In: V.M.Toledo, F. Eccardi, N. Barrera, Carrillo, eds. Introducción a la Ecología Humana (Antologia). Friedrich Ebert Stiftung. México D.F.:179-188.
Herskovits, M. 1987. El Hombre y sus Obras. Fondo de Cultura Económica. México, D.F..

Juárez, C. 1988. Análisis del incremento periódico de caoba (Swietenia macrophylla King) y cedro (Cedrela odorata) en un relicto de selva en el estado de Campeche. Tesis, Universidad Autónoma de Chapingo, Chapingo, México. 88 p.

Keyes, M. 1995. Estudios de postgrado forestal tropical. La ciencia y el hombre 19: 29-52.

Konrad, H. 1988. Tropical forest policy and practice during the Mexican Porfiriato, 1876-1910. Journal of Forest History. (En prensa). 13 p.

Lamb, B. 1966. Mahogany of Tropical America: Its Ecology and Management. University of Michigan Press, Ann Arbor, Michigan, E.U.A.

Macario, P. 1991. La repoblación natural en una selva mediana subperennifolia en Quintana Roo bajo aprovechamiento forestal. Tesis, Colegio de Postgraduados, Montecillo, México. 131 p.

Márquez, A. 1943. La explotación del chicle en la región de Tuxpan, Ver. Tesis Profesional, Escuela Nacional de Agricultura, Chapingo, Mexico. 47 p.

Medina, B. 1948. La explotación forestal en el territorio de Quintana Roo. Tesis, Escuela Nacional de Agricultura, Chapingo, Mexico. $67 \mathrm{p}$.

Morley, S. 1981. Agricultura. In: Pérez, A., S. Morley, E. Hernández, L. Varquez y A. Barrera, eds. La milpa en los mayas de Yucatán. Universidad Autónoma de Yucatán, Merida, Yuc.: 29-44. 
Murphy, J. 1992. Integración de la explotación forestal comercial a la agricultura milpera en la zona maya de Quintana Roo. In: Zizumbo, D., C. Rassumssen, L. Arias and S. Tehran, eds. La modernización de la milpa en Yucatan: utopia o realidad. CICY. Mérida, Yuc.:315-324.

Richards, E. 1991. The forest ejidos of south-east México: A case study of community based sustained yield management. Commonwealth Forestry Review, 70 (4):290-312.

Richards, E. 1993. Lessons for participatory natural forest management in Latin America: case studies from Honduras, México and Peru. Journal of World Forest Management, 7:1-25.

Rosado, L. 1940. El cooperativismo en Quintana Roo. In: Careaga V. L. ed. Lecturas Básicas para la Historia de Quintana Roo. Antología. Tomo II. Fondo de Fomento Editorial del Gobierno del Estado de Quintana Roo.

SEDESOL. 1993. Areas naturales protegidas de México. Secretaría de Desarrollo Social México. D.F. 216p.
Secretaria de Educación Pública (SEP). 1985. Quintana Roo. Entre la selva y el mar. Monografía Estatal. México, D.F. 3 Vol.

Snook, L. 1993. Stand dynamics of mahogany (Swietenia macrophylla, King) and associate species after fire and hurricane in the tropical forests of the Yucatan Peninsula, Mexico. Ph.D. Dissertation, Yale University, New Haven, CN, EUA. 254 p.

Tropical Ecosystems Directorate (TDE). 1993.The Maya forest: key issues and recommendations for action. A workshop report. The Central America Commission of Environment and Development. U.S: Man and the Biosphere Program. Flores, Guatemala.

Varela B., H. 1985. Cultura y resistencia cultural: una lectura política. Cultura. Ediciones El Caballito, Secretaría de Educación Pública, México, D.F. 